\title{
Strates
}

STRATES Matériaux pour la recherche en sciences sociales

15 | 2008

Union européenne - Voisinages. La quête d'une intégration régionale

\section{Enjeux, objectifs et instruments d'une intégration régionale agroalimentaire méditerranéenne}

\section{Christian Dubreuil}

\section{OpenEdition}

\section{Journals}

Édition électronique

URL : http://journals.openedition.org/strates/6534

DOI : $10.4000 /$ strates.6534

ISSN : $1777-5442$

Éditeur

Laboratoire Ladyss

Édition imprimée

Date de publication : 1 janvier 2008

Pagination : 85-95

ISSN : 0768-8067

Référence électronique

Christian Dubreuil, «Enjeux, objectifs et instruments d'une intégration régionale agroalimentaire méditerranéenne », Strates [En ligne], 15 | 2008, mis en ligne le 22 janvier 2013, consulté le 08 septembre 2020. URL : http://journals.openedition.org/strates/6534 ; DOI : https://doi.org/10.4000/ strates.6534

Ce document a été généré automatiquement le 8 septembre 2020.

Tous droits réservés 


\title{
Enjeux, objectifs et instruments d'une intégration régionale agroalimentaire méditerranéenne
}

\author{
Christian Dubreuil
}

\section{NOTE DE L'AUTEUR}

Cet article est issu d'un groupe de travail de l'Institut de prospective économique du monde méditerranéen, auquel ont participé en 2006-2007 des spécialistes des questions agricoles et rurales venus de plusieurs pays du Bassin méditerranéen : Jean-Louis Rastoin tout particulièrement ; ainsi que Charles Abdallah, Najib Akesbi, Hassan Benabderrazik, Abdelhamid Bencherif, Felisa Cena, Gonzalo Escribano, Alia Gana, JeanLouis Guigou, Tarik Haddi, Yahia Hamlaoui, Bertrand Hervieu, Guilio Malorgio, Henri Regnault, Tariq Sijilmassi, Marwan Soliman, Yavuz Tekelioglu, Selma Tozanli.

1 Plusieurs événements ou tendances fortes des dernières années favorisent une remise à plat de la question agroalimentaire dans l'ensemble euroméditerranéen. Il y a d'abord des évolutions générales, surtout propres au Nord. Le cycle de Doha de l'OMC vient d'échouer, notamment sur les questions agricoles. Conçu à l'origine comme un cycle de développement intégrant des normes sociales et environnementales dans le commerce mondial, reconnaissant les origines géographiques des productions agricoles, ce cycle s'est vite réduit à un nouveau cycle tarifaire et s'est auto bloqué en raison des intérêts contradictoires des États Unis, de l'Union européenne, des pays émergents et des pays en développement. Il s'agit probablement du dernier cycle multilatéral à base tarifaire ; une relance postérieure à 2009 devra partir sur de nouvelles bases et prendre en compte le besoin de régulation des échanges, indispensable pour ne pas transformer la mondialisation en facteur de destruction des sociétés, et réintroduire dans la négociation la question des normes sociales et environnementales. 
2 Quelle est la place de la Méditerranée dans ce nouveau contexte politique ? Il faut dire que la relance par l'Union européenne des négociations bilatérales à la suite de l'échec $\mathrm{du}$ cycle de Doha concerne paradoxalement beaucoup plus des partenaires lointains (Corée du Sud...) que nos proches voisins du Sud. Sur le plan multilatéral, les pays méditerranéens n'ont pas pu construire de position commune dans les négociations d'une OMC dont quatre pays du partenariat euroméditerranéen (Liban, Autorité Palestinienne, Syrie, Algérie) ne sont pas encore membres. Les pays de la rive sud sont membres de différents groupes (G10, G20, G90) et de ce fait pèsent peu dans les négociations.

3 Il y a ensuite la réforme de la PAC qui, après l'accord de Luxembourg de juin 2003 et le compromis sur le budget européen 2007-2013 de Bruxelles de décembre 2005, continue son évolution, sous l'effet de demandes accrues pour la protection de l'environnement et d'une remise en cause croissante des subventions aux agriculteurs. Les aides directes à la production agricole (premier pilier de la PAC) disparaîtront sous cette forme ou se contracteront très fortement au plus tard en 2013, au bénéfice du deuxième pilier (développement rural). Or ce deuxième pilier est potentiellement applicable à la rive sud de la Méditerranée, que ce soit en termes de méthode ou en termes financiers.

4 Autre élément de ce nouveau contexte, la recherche d'une meilleure sécurité de l'approvisionnement alimentaire pour les États, la sécurité sanitaire des aliments, l'importance accordée à la nutrition, la critique de la «malbouffe » et les progrès de l'obésité ont un impact grandissant dans la détermination des stratégies des entreprises et des politiques publiques dans le secteur. La valorisation du modèle agroalimentaire méditerranéen pourrait constituer une réponse partielle mais forte à ces changements.

5 En ce qui concerne les politiques Euromed enfin, la Politique Européenne de Voisinage («tout partager sauf les institutions ») conduit de nombreux pays du Sud à poser la question agroalimentaire. La conférence de Barcelone de 1995 et le sommet Euroméditerranéen de novembre 2005 ont décidé de libéraliser totalement les échanges industriels d'ici 2010, et partiellement les échanges agricoles en tenant compte d'une certaine « exception agricole ». Cette libéralisation des échanges est nécessaire, car elle aura pour conséquence théorique le démantèlement des systèmes de protection des pays du Sud qui ralentissent la modernisation nécessaire du secteur, l'augmentation des échanges entre les pays de la région, et la spécialisation de ceux-ci dans les biens qu'ils sont capables de produire au meilleur coût. Mais elle n'est pas suffisante, car elle devrait avoir à court terme des effets dévastateurs sur la production agricole des pays de la rive Sud, du fait de la position de force des productions de la rive nord dans le domaines des céréales, des produits laitiers ou de la viande, du fait aussi du dualisme de l'agriculture des pays du Sud entre agriculture traditionnelle et agriculture exportatrice. Cela devrait provoquer l'accélération de l'exode rural vers des villes mal capables d'accueillir de nouveaux migrants, constituant ainsi un facteur de déstabilisation politique majeur.

$6 \quad$ Il faut donc réguler cette phase de transition que constitue l'ouverture des échanges agricoles des pays du Sud de la Méditerranée. C'est une affaire nationale pour chacun de ces pays, c'est aussi une affaire régionale au sens euroméditerranéen du terme. L'agriculture est sans doute le secteur d'activité pour lequel une régulation d'échelle régionale est la plus requise (première partie de cet article), d'autant que le statut dérogatoire du secteur agricole à l'OMC rend possible des propositions innovantes. 
L'objectif de ce texte est de montrer la nécessité et la possibilité d'une régulation régionale de la mondialisation agricole en Méditerranée. Cette régulation passe par une action sur l'offre (soutenir la modernisation des agricultures au Sud) et par une action sur la demande (promotion du modèle agroalimentaire méditerranéen). Elle passe aussi par une approche territoriale indispensable (aménagement du territoire pour amener les infrastructures de base, systèmes productifs localisés et AOC) (deuxième partie). La troisième partie esquisse les instruments de la régulation d'un grand marché agricole euroméditerranéen. C'est une nécessité pour le système agroalimentaire du Sud comme $\mathrm{du}$ Nord de la région, si nous voulons résister aux concurrences extérieures grandissantes et valoriser un modèle méditerranéen qui est autant culturel qu'économique.

\section{Les enjeux agricoles, agroalimentaires et ruraux en Méditerranée}

\section{L'agriculture méditerranéenne : une question aussi culturelle}

7 Le modèle alimentaire méditerranéen, à base de fruits et de légumes, a trois qualités : il est reconnu comme nutritionnellement bénéfique; il est convivial parce qu'il repose sur des pratiques agricoles qui se situent socialement aux antipodes de la grande industrie agroalimentaire; il parle aux consommateurs de nombreux pays du monde. Ce modèle méditerranéen n'est pas seulement alimentaire mais agroalimentaire et même socioculturel: il comporte des relations spécifiques entre producteurs et consommateurs, fondés sur la qualité, la traçabilité, la proximité culturelle et parfois géographique (circuits courts de distribution). Les nouveaux défis du développement durable devraient encore renforcer le rôle de l'agroalimentaire sur la base du concept de filière courte, rapprochant le consommateur des produits et l'industriel de l'agriculteur, valorisant les ressources locales et diversifiant les activités dans l'espace rural. Potentiellement, le modèle méditerranéen devrait pouvoir tirer le meilleur parti de cette nouvelle exigence.

\section{Une double menace}

8 Or ce modèle est menacé de deux façons (Hervieu, 2007). Sur la production d'abord. On sait qu'une concurrence au sein de la région méditerranéenne malmène à la fois les pays de la rive Sud et le "Sud du Nord» (régions méditerranéennes des pays européens), et les pays du Sud entre eux. Par exemple, disparaissent du Liban des productions comme la banane, la pomme ou les fèves menacées de délocalisation sous l'effet des grands programmes agricoles égyptiens. On sait moins que le risque est grand que les productions méditerranéennes prises dans leur ensemble disparaissent sous l'effet de la concurrence australienne, chilienne, argentine, californienne (voir leurs programmes massifs de plantation d'oliviers, pour répondre à une demande mondiale en très forte croissance). Le marché méditerranéen est donc dans une situation paradoxale. Il est potentiellement colossal du fait de ses qualités intrinsèques, mais l'offre n'est pas structurée et même se déstructure. Les habitudes de production et de consommation alimentaires des produits méditerranéens se perdent même en Méditerranée. L'huile d'olive reste un produit de luxe dans bien des pays du Sud. 
9 Du reste, les évolutions en cours, mesurées par les parts de marchés mondiaux, sont défavorables à la rive Nord comme à la rive Sud de la Méditerranée. L'autosuffisance agricole décline en Méditerranée, aux dépens des deux rives (Rastoin et Szedlak, 2006). On pourrait croire que les coûts de transport vont réduire cette concurrence extérieure. Certes, la distance joue au profit d'échanges internes à la région. Il n'y a pas de doute que le coût du fret limitera les effets de l'accord de libre-échange entre le Maroc et les États-Unis par exemple, au profit d'échanges plus locaux. Et à long terme le renchérissement du coût du pétrole ne pourra qu'aller dans le sens d'une régionalisation des échanges. Si l'on ne peut concevoir des échanges purement régionalisés dans tous les secteurs d'activité, on peut tout de même l'imaginer dans le secteur alimentaire où les besoins sont quotidiens et ne peuvent être différés. Mais la vérité oblige à dire que nous pourrions n'atteindre ce moment désirable que dans longtemps, alors que la déstructuration du système agroalimentaire méditerranéen va vite. En outre, les progrès en taille des navires porte containers, l'élargissement du canal de Panama et l'augmentation de capacité des ports comme Le Havre permettent de stabiliser les coûts de transport, au moins à court terme.

10 La menace pèse aussi sur le modèle de consommation. On assiste à l'uniformisation des régimes alimentaires, inspirés des habitudes occidentales (la pizza industrielle, la restauration rapide...) déterminées par le système de production agro-industrielle qui se généralise. Ce modèle passe par de très grandes firmes, de plus en plus spécialisées (Danone a réduit sa production à trois produits et demain sans doute à deux), très financiarisées (poids des fonds de pension), offrant des aliments à des prix faibles en dépit du renchérissement actuel des matières premières agricoles de base, au moyen d'usines de plus en plus importantes produisant des séries qui se comptent en millions d'unités par jour, et offrant, produit par produit, un bon niveau de qualité sanitaire. Mais l'équilibre agroalimentaire nutritionnel est une autre histoire. C'est ce que montrent les débats actuels envisageant d'internaliser les coûts sanitaires du modèle alimentaire dominant ${ }^{1}$. Certes des décisions européennes sont peu envisageables dans ce domaine puisque la fiscalité reste de compétence nationale dans l'Union; mais des orientations générales pourraient être données ${ }^{2}$, qui rencontreraient vraisemblablement l'adhésion des consommateurs de plus en plus sensibilisés aux questions sanitaires puisque les dépenses de santé dépassent désormais celles consacrées à l'alimentation dans le budget des ménages.

\section{Les deux raisons de l'urgence agricole en Méditerranée}

11 La première raison de l'urgence du dossier tient à l'importance du problème de l'emploi. La réduction non maîtrisée de la main-d'œuvre agricole qui résulterait d'une libéralisation "sèche » des échanges agricoles dans la zone de libre-échange Euromed 2010, ajouterait à la masse gigantesque d'emplois que la rive Sud doit déjà créer. Or un système agroalimentaire modernisé est une excellente façon de maintenir et même de développer l'emploi, car l'agroalimentaire comprend l'agriculture mais aussi sa transformation industrielle, la distribution, les industries (équipementiers, machines agricoles...) et les divers services liés (financement, formation, l'ensemble des services concourant déjà pour plus de la moitié de la valeur ajoutée agroalimentaire en France). Contrairement à ce qu'on pense souvent, ce système agroalimentaire, vu de manière large, a conservé un nombre global d'actifs stable sur la rive nord. Il faut bien avoir en tête l'ampleur des transformations à l'œuvre dans la chaîne de valeur, pour mesurer les 
potentialités d'un système agroalimentaire maîtrisé au Sud - et les risques d'un effondrement de l'emploi agricole.

12 La deuxième raison est d'ordre territorial. Dans des pays dont la population est rurale à 30 ou $40 \%$, dans lesquels les croissances démographiques restent fortes (Égypte, Algérie) et dont les densités rurales augmentent encore (Tunisie, Turquie, Maroc...), la déstabilisation agricole aurait des implications territoriales rurales bien entendu mais également urbaines. Ces pays peuvent assumer une légère accélération de l'exode rural, mais maitrisée, or la libéralisation des échanges agricoles pourrait l'accélérer de manière non soutenable. Une politique de développement rural doit donc absolument être mise en place rapidement pour contrebalancer une approche euroméditerrannéenne de l'agriculture jusqu'à présent trop focalisée sur la libéralisation des échanges.

\section{Les objectifs d'une régulation agricole méditerranéenne}

13 La régulation d'un vaste marché agricole euroméditerranéen demanderait des efforts dans plusieurs directions: le soutien à la modernisation des producteurs et une meilleure répartition de la chaîne de valeur entre les deux rives; la promotion du modèle méditerranéen. Sur le plan social et territorial, elle passerait par des aides au revenu peu à peu découplées de la production, des aides à la reconversion et le soutien au développement rural.

\section{Offre : la modernisation du système productif agroalimentaire}

14 Il s'agit d'abord de définir l'avantage comparatif de la Méditerranée. Pour le moment l'agriculture de la région n'a pas de positionnement stratégique. Il faut le trouver et le défendre face à la concurrence mondiale. La Méditerranée ne sait pas produire les produits de base (soja, maïs...) comme le font notamment les pays américains, en revanche les produits méditerranéens doivent être présentés comme sa priorité stratégique.

Deuxièmement, il s'agit d'affirmer et de mettre en œuvre une véritable répartition régionale du travail, tâche sensible qui sera douloureuse pour certains producteurs, mais qui est une nécessité si l'on veut jouer la complémentarité au sein de la région en fonction des potentiels agronomiques et climatiques respectifs (fruits et légumes au Sud, céréales au Nord, pour dire vite). Le modèle de production de fruits et légumes de type Almeria ou Calabre, avec des usages massifs d'eau, de pesticides et de l'emploi de Maghrébins souvent en situation irrégulière et sous payés, n'est pas durable. L'objectif est ici de préférer des complémentarités stratégiques à des productions non durables et/ou à des localisations subies. Dans un tel schéma, la rive nord de la Méditerranée verra disparaitre certaines productions comme les légumes industriels ; mais cela serait compensé par le développement de productions à plus forte valeur ajoutée et de fonctions de service (organisation, marketing, logistique...). Côté Sud, le maintien, par des protections douanières et des subventions massives, de la céréaliculture au Sud est rarement optimal. Évidemment, il ne faut pas sous-estimer l'ampleur du défi : au Maroc 
par exemple, plus d'un million des 1,4 million d'exploitations, soit 6 à 7 millions de personnes, produit des céréales!

Il s'agit, ensuite, de favoriser la descente au Sud de la première transformation liée aux productions primaires. Les industries de troisième et quatrième transformations se localisent près des lieux de consommation, notamment près des nœuds logistiques; il faudra beaucoup de temps pour qu'elles accompagnent, au Sud, l'essor des marchés intérieurs. Raison de plus pour prendre un parti clair sur la première transformation des productions agricoles, qui doivent se développer sur la rive Sud de manière préférentielle - à condition bien sûr que les conditions économiques de la production industrielle y soient modernisées.

Il s'agit enfin de soutenir l'organisation des milieux professionnels au Sud, afin de faire des agricultures du Sud des activités productives - sans tomber dans le productivisme. Cela passe par la mise en réseau des PME et TPE à des fins de partage des coûts, par le soutien aux mesures de restructuration foncière, par le soutien aux organismes interprofessionnels (organisation des filières, interprofessions, instituts techniques, coopératives...). Grâce à quoi ces milieux professionnels tout à la fois :

- assureraient leur modernisation et leur mise aux normes. La production d'huile d'olive espagnole a montré les effets bénéfiques d'une telle modernisation, enrayant son déclin et amorçant une reconquête commerciale spectaculaire ${ }^{3}$;

19 - se renforceraient vis-à-vis du pouvoir des multinationales de l'alimentaire et de la grande distribution ${ }^{4}$;

20 - se mettraient en réseau pour partager les ressources rares et chères et les compétences (par exemple des cadres bien formés pour donner à plusieurs exploitations un directeur de la qualité ou du marketing commun). Le partenariat d'entreprise peut aussi se faire avec les pays du Nord, comme entre les États-Unis et le Mexique ${ }^{5}$.

\section{Demande : la valorisation du modèle alimentaire méditerranéen}

21 Avec le vieillissement de la population, le souci de traçabilité et de qualité sanitaire progresse. La Méditerranée tient là un modèle alternatif, qu'il faudrait défendre et valoriser avec constance - un modèle agroalimentaire méditerranéen défini par la demande et non pas déterminé par les structures productives en cours de concentration. Ce point de vue repose sur deux éléments : d'abord, pour mieux se nourrir, on peut penser que le consommateur accepte de payer plus cher son alimentation; d'autant, ensuite, que l'internalisation des coûts sanitaires pourrait peu à peu renchérir le modèle agroalimentaire dominant. Sur cette base, l'agriculture méditerranéenne pourrait entamer une reconquête de son marché intérieur. C'est évidemment une œuvre de longue haleine, il s'agit de la lancer. On sait que l'opinion publique et les normes générales de santé comme de respect de l'environnement, ont un impact sur les multinationales. Certes il n'existe que peu d'associations de consommateurs au Sud. Mais l'information circule tout de même, ne serait-ce qu'à travers les médias au Sud comme au Nord de la Méditerranée. Elle produit ses effets. 


\section{Territoires : l'approche indispensable}

L'approche territoriale comporte trois aspects liés. Le premier tient à la labellisation des productions méditerranéennes, qu'il s'agirait de négocier à Bruxelles et de faire reconnaître par l'OMC. Il s'agit d'un combat car les libéraux dogmatiques et les ÉtatsUnis préfèrent la libre circulation des marques, c'est-à-dire de produits banalisés par leur contenu mais identifiés par leurs marques imposées par la publicité et circulant sans entraves. Bien entendu la labellisation a une dimension commerciale générale; mais elle a aussi et surtout une dimension territoriale, car c'est sur l'ancrage territorial que repose la qualité des produits de la région méditerranéenne. Par définition, l'activité agricole est liée au territoire. Elle est créatrice de valeur économique et d'emploi, intrinsèquement et par les effets d'entraînement en amont et en aval. Elle est aussi fondatrice d'une culture spécifique et de valeurs sociétales originales dans un monde qui se globalise. Ces différents attributs légitiment une protection juridique du lien entre produits et territoires, et constituent le fondement de la politique européenne des Indications géographiques ${ }^{6}$. Or à l'exception de la Turquie qui dispose d'une réglementation de cette nature et qui a protégé 23 produits agroalimentaires (fruits secs, lentilles, ail, fromage de chèvre, loukoums, marrons glacés), aucun des pays de la rive sud n'a mis en place une protection de type IG, même si des initiatives se font jour comme par exemple l'huile d'argan au Maroc, les dattes en Algérie ou l'agneau en Tunisie. Une Appellation "ombrelle », qui pourrait être "Produits méditerranéens ", pourrait par surcroît coiffer l'ensemble de ces appellations et donner du poids à un marketing mondial.

Le deuxième volet concerne le rôle des sociétés civiles, qui doivent intervenir dans la mise à niveau des filières. Les sociétés civiles sont une condition de la mise en place de Systèmes de production localisés, qui regrouperaient les petites ou moyennes exploitations et entreprises de transformation sur le modèle des clusters et districts de la rive nord. L'Italie a montré la voie avec ses groupes locaux d'action qui associent les entreprises et les collectivités locales. L'engagement des sociétés civiles permettrait d'abord de mieux maîtriser l'amont des filières courtes locales. La meilleure traçabilité possible, c'est celle du contrôle social de la production. L'histoire des appellations contrôlées en Europe est l'histoire de l'organisation locale des producteurs locaux pour contrôler leurs cahiers des charges, pour des coûts accrus mais mutualisés, et avec un bénéfice énorme. La création d'agences indépendantes d'évaluation de la qualité éviterait les abus de cette auto organisation locale. L'engagement des sociétés civiles permettrait aussi une meilleure maîtrise des filières à l'aval : meilleure articulation producteurs-consommateurs, que ce soit par la vente directe ou via les Associations de maintien de l'agriculture paysanne ${ }^{7}$; utilisation des marchés publics (restauration collective...) pour favoriser les produits locaux de qualité.

Le troisième volet concerne un problème qui se pose à peu près dans les mêmes termes au Nord et au Sud: le développement rural. Au Nord, le développement rural, simple politique d'accompagnement lors de la réforme de la PAC de 1992, est devenu une politique à part entière dans la réforme de 1999, confortée par le transfert des aides du premier pilier dans la réforme de 2003. Au Sud, l'adoption par la Turquie d'une stratégie nationale du développement rural ou le programme de renouveau rural algérien montrent la pertinence de ce type de politique. En ce qui concerne le secteur agricole, c'est d'abord le moyen de maintenir une agriculture dans des zones en 
difficulté, par un aménagement du territoire qui apporterait aux espaces ruraux les infrastructures et services de base (routes, électricité, télécoms, écoles, dispensaires) sans lesquels nul développement territorial n'est envisageable. En ce qui concerne l'espace rural, c'est le développement de l'artisanat voire de l'industrie : les ateliers de production déconcentrés et compétitifs qui se sont développés dans bien des campagnes tunisiennes lorsqu'elles sont reliées au système productif urbain, peuvent servir d'exemple. C'est aussi la diversification vers le tourisme, les activités liées à la résidence des actifs et des retraités de la rive nord. Ce sont toutes les actions de promotion de l'emploi et notamment de l'emploi féminin, via le microcrédit par exemple, en forte croissance dans le monde et qui est très territorialisé, ou à travers tous types de projets de développement local.

\section{Les instruments de la régulation}

\section{Les instruments commerciaux}

La libéralisation des échanges euroméditerranéens doit être modulée : elle doit être progressive et asymétrique (le Nord ferait plus que le Sud). Sa première étape serait l'extension de la préférence communautaire à l'ensemble de la Méditerranée. Cela suppose le respect de la règle du jeu par le Nord, afin de rendre possible les exportations de produits du Sud qui ont fait l'objet d'investissements de modernisation mais se voient refuser de fait (barrières non tarifaires) les débouchés qu'ils seraient en droit d'attendre au Nord. Cela suppose aussi la réciprocité : le Sud devrait, lui aussi, accepter la notion de préférence - ce qui soulève par exemple le problème de l'accord de libre-échange entre le Maroc et les États-Unis. Au Nord comme au Sud, l'intégration régionale a un prix.

La deuxième étape serait l'établissement de règles communes dans deux ou trois filières, afin de constituer des organisations euroméditerranéennes communes de marché. Si l'on veut susciter des complémentarités motivantes entre acteurs locaux et régionaux, il faut un cadre facilitateur du dialogue, encourageant des relations entre agriculture, industrie et commerce ; la mise en place d'une Organisation Commune de Marché «fruits et légumes » euroméditerranéenne, en particulier, pourrait y répondre.

\section{Les instruments financiers}

Il faut imaginer un programme de financement sur vingt ans au moins. Les propositions d'aides au revenu des agriculteurs du Sud sur une base majoritairement européenne avec contrepartie nationale des pays du Sud, ou d'aides à la reconversion, sont des propositions déjà anciennes. Il suffit de passer aux actes.

Une première question est celle de l'estimation des coûts. L'exemple des céréaliculteurs au Maroc donne une fourchette entre 0,5 milliard d'euros annuels (si l'on limite les subventions aux dix premiers quintaux pour éviter d'entrer dans une logique productiviste) et 2 milliards (salaire minimum marocain pour un million de ménages). Ces sommes ne sont pas gigantesques mais d'un côté elles sont très supérieures au total actuel des subventions européennes dans le cadre de MEDA (acronyme de « Mesures d'ajustement») et de l'autre les priorités de l'UE vont aux nouveaux États-membres puis aux futurs États-membres (Balkans, Turquie). 
29 La Politique de voisinage au Sud devrait donc se centrer de manière forte sur l'agriculture. S'il n'est pas raisonnable de créer un nouvel instrument financier, il est envisageable d'utiliser les instruments existants. Une solution pourrait venir de ce que l'on pourrait appeler la «méditerranéisation » de la PAC : à la fois sur le premier pilier (un soutien accru aux productions méditerranéennes ${ }^{8}$ ), et surtout sur le deuxième pilier dont on a dit qu'il est appelé à se développer. Il y a des avantages objectifs convergents entre les pays méditerranéens européens et de la rive sud à valoriser le deuxième pilier de la PAC : c'est un moyen de sauvegarder un soutien financier à l'agriculture en mal de légitimation, et c'est un moyen de ne plus faire apparaître la PAC comme une forteresse pour le Nord. En particulier, on pourrait étendre le programme «Leader» au Sud. Du reste, «Leader Med: un territoire, un projet, un contrat » est envisagé depuis deux ans par les ministres de l'agriculture des deux rives9.

Une autre approche serait l'établissement d'un Fonds agricole euroméditerranéen cofinancé (même si principalement financé par l'UE) qui financerait notamment les volets agricoles des Plans d'action de la PEV (modernisation des structures productives agricoles infrastructures de base; sorte d'équivalent du Fonds de cohésion de l'Espagne, du Portugal ou de la Grèce naguère, mais en ajoutant le financement des services de base du développement humain aux routes et à l'électrification).

31 En tout état de cause, il faudra aussi trouver des financements au Sud, que ce soit pour des raisons financières (l'argent de Bruxelles ne suffira pas) ou pour des raisons politiques (partenariat Nord-Sud, responsabilisation des acteurs du Sud, engagement de leurs ressources financières nationales aux dépens des politiques classiques de subventionnement ou du tout hydraulique). Or tous les pays du Sud ne disposent pas des ressources de l'État Algérien. Indispensable, le cofinancement soulève le problème des pays déjà surendettés. Une des solutions est à chercher du côté de la sousfiscalisation des agriculteurs, dont bénéficient même les plus riches d'entre eux.

\section{Les instruments politiques}

32 S'agissant des instruments politiques explicitement dédiés à l'espace Euromed, la Politique Européenne de Voisinage est le vecteur à utiliser en priorité. Si tous les pays du Sud ne sont pas partants, cette politique autorise des rythmes nationaux différents. Les Plans d'Action peuvent très bien être le cadre d'emprunt des méthodes qui ont fait leurs preuves au Nord, en particulier pour le deuxième pilier de la PAC.

Cela dit, deux observations complémentaires méritent d'être faites. La première est que, dans le domaine de la coopération euroméditerranéenne, il est impératif que les priorités soient définies à l'écoute des pays du Sud et en particulier des acteurs professionnels. Trop souvent en effet, les programmes de coopération bilatéraux sont définis par des experts du Nord, sans réelle connaissance des réalités du terrain. La seconde observation est que la PEV n'offre pas le cadre Méditerranéen d'analyse et d'action qui caractérisait les accords MEDA de 1995. En devenant bilatérale (UE/chaque pays de la rive Sud), la PEV est devenue plus efficace, mais elle a aussi perdu cette dimension régionale méditerranéenne indispensable à la promotion d'un modèle agroalimentaire commun. La juxtaposition des Plans d'Action d'une durée de trois à cinq ans déjà signés avec le Maroc, la Tunisie, la Jordanie, l'Autorité Palestinienne ou Israël, ne permet pas de donner de la visibilité à la question agricole. Reste à savoir si le 
projet d'Union Méditerranéenne lancée par le gouvernement français en 2007 sera de nature à donner à l'agriculture cette priorité nécessaire.

\section{Conclusion}

Inscrire l'agriculture - et au-delà d'elle l'ensemble du système agroalimentaire - des pays du Sud et leurs territoires ruraux dans la modernité, tel est l'objectif du "Pacte agricole et agroalimentaire méditerranéen " qui rassemble les préconisations de l'IPEMed (2007), à la suite des premières propositions de Lorca et Escribano (1999). Ce pacte a peu à voir avec une simple zone de libre-échange. C'est plutôt un ensemble de règles et une mutualisation qui permet à chacune des agricultures des deux rives de tirer partie de ses avantages, de bénéficier de la dimension de la région méditerranéenne et de ses complémentarités, dans le contexte d'une concurrence globale accrue. Un tel pacte offrirait aux agriculteurs des marchés régulés, un cadre institutionnel et normatif, une protection extérieure, un label mondialement reconnu, un instrument de reconquête de marchés intérieurs pour les produits méditerranéens, un instrument de développement équilibré de l'espace rural. En contrepartie il imposerait aux agriculteurs de deux rives les règles de l'acquis communautaire: sécurité sanitaire des aliments, protection de l'environnement. Et il supposerait que soit politiquement acceptée, de part et d'autre, la rationalité économique régionale : complémentarité des productions et de leurs calendriers, partage de la valeur ajoutée entre les deux rives.

\section{BIBLIOGRAPHIE}

Escribano G., Lorca A., 1999, « Vers un pacte agricole méditerranéen? », Groupe d'études et de recherche sur la Méditerranée, annuaire de la Méditerranée, Paris, Publisud, publié dans FEMISE, Rapport 2003, La libéralisation agricole et le partenariat euroméditerranéen, Marseille.

Hervieux B. (dir.), 2007, AgriMed, Agriculture, pêche, alimentation et développement rural durable dans la région méditerranéenne, Rapport 2006, Montpellier, CIHEAM.

IPEMed, 2007, « Un Pacte agricole et agroalimentaire méditerranéen », polygr., http// www.ipemed.coop/

Rastoin J.-L., Szedlak A., 2006, Dynamique des échanges internationaux agricoles et agroalimentaires de la zone euroméditerranéenne, UMR Moisa/IPEMed - Crédit Agricole du Maroc, Paris, en ligne sur http://www.ipemed.coop/

\section{NOTES}

1. Les États-Unis eux-mêmes envisagent d'imposer une fat tax. 
2. C'est le sens de l'action de l'association internationale "Slow Food " qui veut favoriser la gastronomie, les produits bons au plan des caractéristiques organoleptiques, propres au plan environnemental et justes au plan de la rémunération du producteur.

3. Au passage, disons que cette mise aux normes internationales n'est pas liée aux seuls échanges internationaux : des productions vouées au marché intérieur bénéficieraient tout autant d'une telle modernisation. Il existe d'ailleurs de vrais problèmes d'intoxication alimentaire au Sud, la sécurité sanitaire des aliments doit y être une priorité.

4. Aux Pays-Bas par exemple, les producteurs de fleurs se sont regroupés en une seule entité pour mieux négocier avec les distributeurs.

5. Le mouvement de concession de terres agricoles à des investisseurs étrangers observé dans le Maghreb peut constituer, s'il est bien articulé avec le contexte local et les filières agroalimentaires, un puissant levier de modernisation et de développement économique régional.

6. C'est le triptyque, défini en 1992, AOP (Appellation d'origine protégée), IGP (Indication géographique protégée), STG (Spécialité traditionnelle garantie).

7. Les AMAP qui connectent consommateurs locaux et agriculteurs locaux par des accords de distribution préférentielle, croissent rapidement dans les pays industrialisés.

8. Rappelons que l'ensemble de la viticulture européenne reçoit de la PAC 1,5 milliard d'euros, les fruits et légumes 2 milliards, quand $57 \%$ des 37 milliards vont aux grandes cultures et $27 \%$ aux viandes bovines.

9. Les ministres ont demandé une expérimentation, des projets pilotes. Des banques de projets de développement existent, elles ont leurs porteurs de projets. Elles n'attendent que le financement, voilà l'obstacle.

\section{RÉSUMÉS}

L'article définit ce que pourrait être un pacte agroalimentaire euroméditerranéen, ménageant une transition maîtrisée vers une libéralisation complète des échanges. Cela passe par une action sur l'offre : il faut soutenir la modernisation des agricultures au Sud, jouer la complémentarité agro-climatique au sein de la région Euromed, et mieux répartir la chaîne de la valeur entre rive Nord et rive Sud - c'est-à-dire préférer des complémentarités stratégiques à des localisations subies et favoriser la descente au Sud de la première transformation liée aux productions primaires. Cela passe aussi par une action sur la demande à travers la promotion du modèle agroalimentaire méditerranéen, pour des raisons à la fois nutritionnelles, de santé publique, et de compétition qu'imposent les pays producteurs extérieurs à la région. Cela passe enfin par une action territoriale indispensable, que ce soit en matière d'aménagement du territoire pour amener les infrastructures de base encore trop souvent absentes, en matière d'Indications Géographiques et de systèmes productifs localisés sachant mobiliser les sociétés civiles vis-à-vis du pouvoir des multinationales de l'alimentaire et de la grande distribution, ou en matière de développement rural selon des méthodes qui ont porté leurs fruits sur la rive Nord à travers le deuxième pilier de la PAC.

This paper proposes what could become a Euromediterranean agribusiness pact, paving the way to a controlled liberalisation of the commercial exchanges of agricultural goods. On the one hand, such a pact should be based on a "supply oriented" policy following three main directions : 
to support the modernisation of the agriculture in the countries of the south bank; to take advantage from the agricultural and climatic complementarities of both banks of the Euromed region; to improve the geographical distribution of the value chain on both sides of the Medtierranean. Such a policy would encourage the resettlement of the first transformation of agricultural goods on the south bank, for example. On the other hand, this policy should be a "demand oriented" one which should promote the Mediterranean agribusiness model, taking account of nutritional reasons, of public health problems and of the increasing competition with agricultural goods imported form other countries. Last, the author addresses the territorial dimension of such a policy, with a special effort for the development of basic infrastructures and of relevant Geographic Indications. He recommends the emergence of local productive systems and the mobilization of local actors against the pressure of transnational firms and department stores chains. Last, as far as the spatial development issue is concerned, the author proposes to transfer some methods of the common agricultural policy to the territory of the Mediterranean third countries.

\section{INDEX}

Index géographique : Europe, Méditerranée

Mots-clés : rapports Nord-Sud, politique agricole, développement rural, commerce agroalimentaire

\section{AUTEUR}

\section{CHRISTIAN DUBREUIL}

Inspecteur général de l'Agriculture 